\title{
Identification of a Novel Fusion Gene, IRF2BP2-RARA, in Acute Promyelocytic Leukemia
}

\author{
C. Cameron Yin, MD, PhDa,,; Nitin Jain, $\mathrm{MD}^{\mathrm{b}, *}$; Meenakshi Mehrotra, PhD ${ }^{\mathrm{a}, *}$; Jianhua Zhang, $\mathrm{PhD}$; \\ Alexei Protopopov, PhDc; Zhuang Zuo, MD, PhD; ; Naveen Pemmaraju, MD ${ }^{b}$; Courtney DiNardo, MD $^{\text {b }}$; \\ Cheryl Hirsch-Ginsberg, MD; Sa A. Wang, MDa; L. Jeffrey Medeiros, MDª Lynda Chin, MDc; \\ Keyur P. Patel, MD, $\mathrm{PhD}^{\mathrm{a}}$; Farhad Ravandi, $\mathrm{MD}^{\mathrm{b}}$; Andrew Futreal, $\mathrm{PhD}^{\mathrm{c}}$; and \\ Carlos E. Bueso-Ramos, MD, PhDa
}

\begin{abstract}
Acute promyelocytic leukemia (APL) is characterized by the fusion of retinoic acid receptor alpha (RARA) with promyelocytic leukemia $(P M L)$ or, rarely, other gene partners. This report presents a patient with APL with a novel fusion between RARA and the interferon regulatory factor 2 binding protein 2 (IRF2BP2) genes. A bone marrow examination in a 19-year-old woman who presented with ecchymoses and epistaxis showed morphologic and immunophenotypic features consistent with APL. PML oncogenic domain antibody was positive. Results of fluorescence in situ hybridization, conventional cytogenetics, reverse transcription-polymerase chain reaction (RT-PCR), and oligonucleotide microarray for PML-RARA and common APL variant translocations were negative. Next-generation RNA-sequencing analysis followed by RT-PCR and direct sequencing revealed distinct breakpoints within IRF2BP2 exon 2 and RARA intron 2. The patient received all-trans retinoic acid, arsenic, and gemtuzumab ozogamicin, and achieved complete remission. However, the disease relapsed 10 months later, 2 months after consolidation therapy. This is the first report showing involvement of IRF2BP2 in APL, and it expands the list of novel RARA partners identified in APL. (J Natl Compr Canc Netw 2015;13:19-22)
\end{abstract}

From the aDepartments of Hematopathology, 'Leukemia, 'Genomic Medicine, and dLaboratory Medicine, The University of Texas MD Anderson Cancer Center, Houston, Texas.

*These authors contributed equally to this work.

Submitted June 24, 2014; accepted for publication September 12, 2014.

Dr. Ravandi has disclosed that he receives research support from Sunesis Pharmaceuticals, Inc.; Bristol-Myers Squibb Company; Novartis AG; Seattle Genetics, Inc.; Merck \& Co., Inc.; and Actinium Pharmaceuticals, Inc. He is on the advisory board for Sunesis Pharmaceuticals, Inc.; Amgen Inc.; Seattle Genetics, Inc. He receives honoraria from Sunesis Pharmaceuticals, Inc.; Seattle Genetics, Inc. The remaining authors have disclosed that they have no financial interests, arrangements, affiliations, or commercial interests with the manufacturers of any products discussed in this article or their competitors.

Corresponding authors: Carlos E. Bueso-Ramos, MD, PhD, Department of Hematopathology, The University of Texas MD Anderson Cancer Center, 1515 Holcombe Blvd, Houston, TX 77030. E-mail: cbuesora@mdanderson. org

Andrew Futreal, PhD, Department of Genomic Medicine, The University of Texas MD Anderson Cancer Center, 1515 Holcombe Blvd, Houston, TX 77030. E-mail: afutreal@mdanderson.org
Acute promyelocytic leukemia (APL) is commonly associated with $\mathrm{t}(15 ; 17)(\mathrm{q} 24.1 ; \mathrm{q} 21.2)$, which results in the fusion of the promyelocytic leukemia (PML) gene at chromosome 15q24.1 with the retinoic acid receptor alpha (RARA) gene at chromosome 17q21.2. ${ }^{1}$ This fusion accounts for the disease response to all-trans retinoic acid (ATRA), and patients with APL usually have a favorable prognosis. ${ }^{1}$ However, although rare, RARA fuses with other genes in patients with APL, and reported partner genes include zinc finger and BTB domain containing 16 (ZBTB16, also known as promyelocytic leukemia zinc finger, PLZF), ${ }^{2,3}$ nucleophosmin (NPM1), ${ }^{4}$ nuclear mitotic apparatus (NUMA1), ${ }^{5}$ signal transducer and activator of transcription 5B (STAT5B), ${ }^{6}$ cAMP-dependent protein kinase type I-alpha regulatory subunit (PRKAR1A), ${ }^{7}$ FIP1-like 1 (FIP1L1), ${ }^{8}$ BCL6 corepressor (BCOR), ${ }^{9}$ and oligonucleotide/oligosaccharide-binding fold-containing $2 \mathrm{~A}(\mathrm{OBFC} 2 \mathrm{~A}){ }^{10}$ The identity of the partner gene is clinically important, because some RARA partner genes have been associated with resistance to ATRA therapy, in particular ZBTB16-RARA and STAT5B-RARA.3,6 This report presents a patient with APL for whom cytogenetic and molecular testing did not show evidence of $\mathrm{t}(15 ; 17)(\mathrm{q} 24.1 ; \mathrm{q} 21.2) / P M L-R A R A$, which led to the discovery of interferon regulatory factor 2 binding protein 2 (IRF2BP2), a novel gene partner for RARA.

\section{Case Report}

A 19-year-old woman presented with ecchymoses and epistaxis. Results of a CBC count showed a WBC count of $4.5 \times 10^{3} / \mathrm{mcL}$, hemoglobin level of $9.1 \mathrm{~g} / \mathrm{dL}$, and a platelet 
count of $29,000 \times 10^{3} / \mathrm{mcL}$, with $28 \%$ promyelocytes. She had a prolonged prothrombin time (PT) and activated partial PT (aPTT) of 19.6 and 46.3 seconds, respectively, an elevated D-dimer level greater than $20 \mathrm{mcg} / \mathrm{mL}$, and a decreased fibrinogen level of $60 \mathrm{mg} /$ $\mathrm{dL}$. Promyelocytes in the peripheral blood and disseminated intravascular coagulopathy were suggestive of APL. The patient was initiated on ATRA therapy. Her bone marrow was hypercellular (100\%), with 59\% promyelocytes, infrequent Auer rods, strong myeloperoxidase reactivity (cytochemistry), and a positive PML oncogenic domain (POD) test ${ }^{11}$ (Figure 1). Flow cytometry immunophenotyping showed an aberrant promyelocytic immunophenotype. Conventional cytogenetic analysis showed a diploid karyotype. Fluorescence in situ hybridization using a break-apart RARA probe was negative. Reverse transcription-polymerase chain reaction (RT-PCR) and oligonucleotide microarray did not detect any translocations commonly seen in APL including $\mathrm{t}(15 ; 17)(\mathrm{q} 22 ; \mathrm{q} 21) / P M L-R A R A$, $\mathrm{t}(5 ; 17)(\mathrm{q} 35 ; \mathrm{q} 21) / N P M 1-R A R A, \quad \mathrm{t}(11 ; 17)(\mathrm{q} 13 ; \mathrm{q} 21) /$ NUMA1-RARA, and $\mathrm{t}(11 ; 17)(\mathrm{q} 23 ; \mathrm{q} 21) / 2 B T B 16-$ RARA. ${ }^{12}$ A screen for gene mutations detected a lowlevel mutation in NRAS (c.35G>A, p.Gly12Asp, $<5 \%)$.

Based on the morphologic features and positive myeloperoxidase and POD tests, the patient was diagnosed with APL. Therapy with ATRA was continued, and arsenic trioxide (given on day 2) and gemtuzumab ozogamicin ( 1 dose on day 6 ) were added to the therapeutic regimen. ${ }^{13}$ The patient responded to treatment well; however, it took nearly 2 weeks for her PT, aPTT, and fibrinogen levels to return to normal, and her D-dimer level remained slightly elevated at $3.57 \mathrm{mcg} / \mathrm{mL}$ at last checkup, 11 months postdiagnosis of APL. She achieved complete molecular remission 5 weeks later, and received 8 months of consolidation therapy with ATRA and arsenic trioxide. However, the patient experienced relapse 2 months after completion of consolidation therapy. She then received salvage therapy with ATRA, arsenic trioxide, and idarubicin, followed by haploidentical bone marrow transplantation. At the time of writing, she was experiencing complete remission 15 months after the initial diagnosis of APL.

Next-generation RNA-sequencing (RNA-seq) analysis was performed to identify the fusion gene partner. Library construction was performed using 700 ng of total RNA and the TruSeq RNA Sample Prep
Kit v2 (Illumina, San Diego, CA). The library was selectively enriched by 12 cycles of PCR, followed by size selection, per the manufacturer's protocol. The resulting size-fractionated library was sequenced using a 75-base pair (bp) paired-end reads protocol on an Illumina HiSeq 2000 Sequencing System. The resultant *.bcl files were converted to .FASTQ files using Illuminas' offline base calling software CASAVA (http://support.illumina.com/sequencing/sequencing_software/casava.ilmn). After standard quality control using RNA-SeQC (http://www.broadinstitute.org/cancer/cga/rna-seqc), FASTQ files were processed using TopHat-Fusion (http://ccb.jhu.edu/ software/tophat/index.shtml) to detect potential fusions. Supporting evidence (number of split reads, mate pairs, mate pairs with split ends) was manually examined to remove false-positive calls. RNA-seq data from bone marrow aspirate were analyzed for fusion transcripts involving RARA. This resulted in the identification of 87 sequence-unique mate-pair reads that defined a translocation joining intron 1 of IRF2BP2 gene on chromosome 1 to intron 2 of RARA gene on chromosome 17. Sixteen sequenceunique split-reads (spanning putative breakpoints) were identified that further confirmed and refined
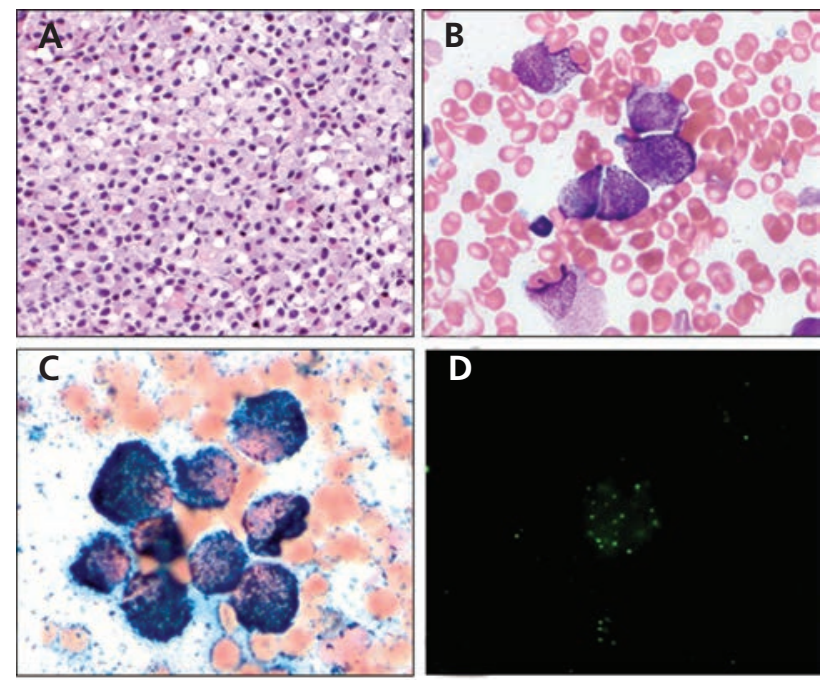

D

Figure 1 Morphologic findings. (A) Bone marrow biopsy specimen showing sheets of leukemic cells with irregular nuclei and abundant eosinophilic cytoplasm (hematoxylin-eosin, original magnification $\mathrm{x} 200$ ). (B) Bone marrow aspirate smear showing promyelocytes with distinct cytoplasmic granules and infrequent Auer rods (Wright-Giemsa stain, original manification $\times 1000)$. (C) Promyelocytes are strongly positive for myeloperoxidase (original magnification x1000). (D) Promyelocytic oncogenic domain antibody shows that the promyelocytes are positive (original magnification $\times 1000$ ). 
the IRF2BP2-RARA fusion. The data suggest that multiple transcripts are likely driven off the fusion. Because of the strength of the RNA-seq data and the involvement of RARA, a novel IRF2BP2-RARA fusion was likely involved in the pathogenesis of this case of APL (Supplemental Table 1, available online, in this article, at JNCCN.org).

RT-PCR and genomic DNA PCR was performed to detect the IRF2BP2-RARA fusion. Total RNA was reverse transcribed using random hexamers. PCR was performed using primers designed to amplify IRF2BP2-RARA fusion transcripts from cDNA and genomic DNA (Figure 2A, B). The PCR product was sequenced and analyzed using the EMBL-EBI multiple sequence alignment tool Clustal Omega (Hinxton, Cambridgeshire, UK). Amplicons were obtained of $310 \mathrm{bp}$ and $323 \mathrm{bp}$, respectively (Figure 2C). Sanger sequencing of genomic DNA PCR products revealed that the IRF2BP2-RARA fusion involved exon 2 of IRF2BP 2 and intron 2 of RARA with breakpoints at position $1687 \mathrm{bp}$ in IRF2BP2 and 41,620 bp in RARA (Figure 2D, E). These findings confirm the fusion partners and breakpoints detected by RNA sequencing.

\section{Discussion}

IRF2BP2, located at chromosome 1q42.3, encodes a nuclear protein that contains an $N$-terminal zinc finger and a C-terminal RING finger domain that interacts specifically with the C-terminal transcriptional repression domain of IRF2. ${ }^{14}$ IRF2BP2 acts as a transcriptional corepressor and represses transactivation of nuclear factor of activated T cells (NFAT) that regulates genes involved in cell cycle, differentiation, and apoptosis. ${ }^{15}$ IRF2BP2 is also an ischemia-induced coactivator of vascular endothelial growth factor A expression, which may contribute to revascularization of ischemic muscle. ${ }^{16}$ IRF2BP2 is a direct target gene of TP53, and its overexpression inhibits apoptosis by impeding TP53-mediated transactivation of the TP21 and BAX genes. ${ }^{17}$ IRF2BP2 has been identified as a antiapoptotic factor in breast cancer cell lines, ${ }^{18}$ and a tumor-associated antigen in monoclonal gammopathy of undetermined significance. ${ }^{19}$ Whole transcriptome sequencing identified a novel IRF2BP2-CDX1 fusion as a result of $\mathrm{t}(1 ; 5)(\mathrm{q} 42 ; \mathrm{q} 32)$ in a case of mesenchymal chondrosarcoma. ${ }^{20}$

RARA functions by binding to retinoic acid response elements as a heterodimer with retinoid

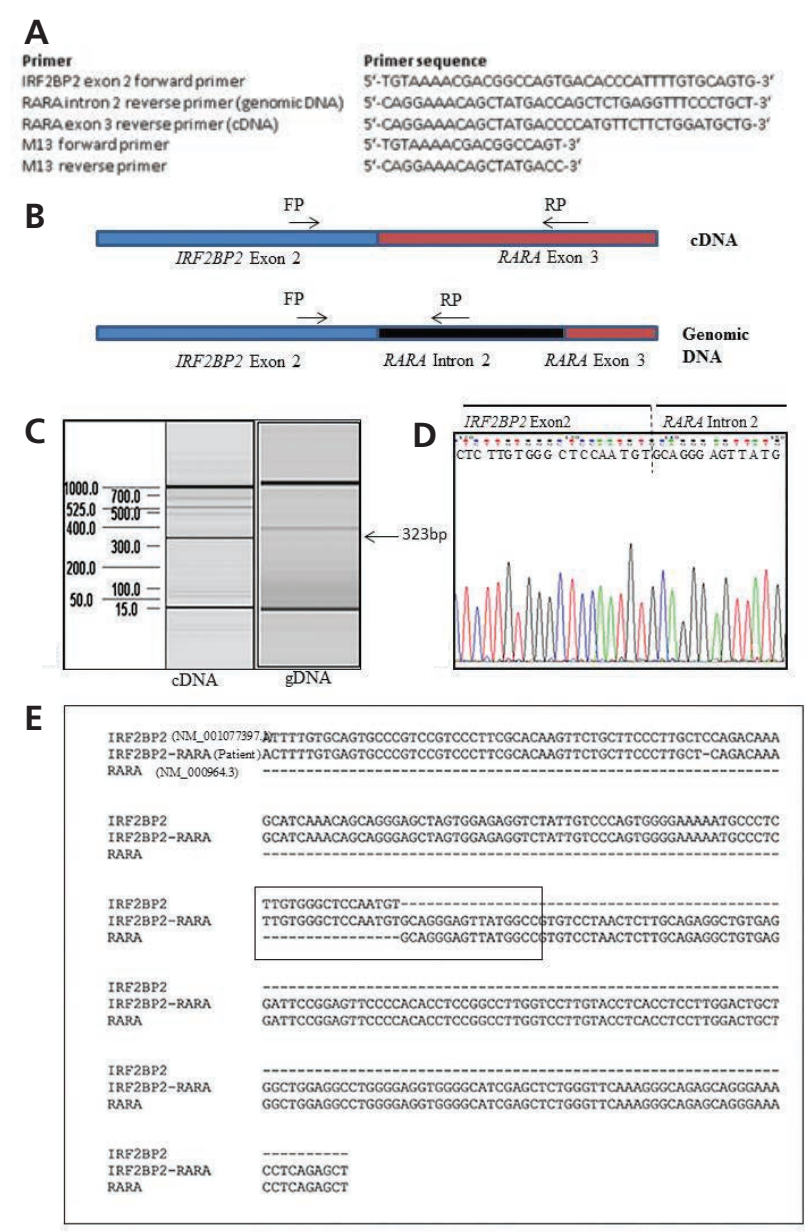

Figure 2 Molecular characterization of IRF2BP2 and RARA fusion. (A) Primer sequences for the detection of IRF2BP2-RARA fusion. Primers were designed in exonic and intronic regions of the IRF2BP2 and RARA genes to cover putative fusion sequences from CDNA and genomic DNA. To facilitate sequencing of PCR amplicons, primers were tagged with $M 13$ universal sequences. (B) Primer design covering IRF2BP2 exon 2 and RARA exon 3 or intron 2 for detection of IRF2BP2RARA fusion in CDNA and genomic DNA, respectively. (C) Results of reverse transcription-polymerase chain reaction (RT-PCR) and genomic PCR for detecting IRF2BP2-RARA fusion. RT-PCR using forward primer (FP) from IRF2BP2 exon 2 and reverse primer (RP) from RARA exon 3 gives rise to an amplicon of 310 base pairs (bp). Genomic PCR using FP from IRF2BP2 exon 2 and RP from RARA intron 2 gives rise to an amplicon of $323 \mathrm{bp}$. (D) Direct sequencing of genomic PCR products reveal a IRF2BP2-RARA fusion with a distinct breakpoint and part of sequences from IRF2BP2 exon 2 and RARA intron 2. (E) Alignments of genomic sequences of IRF2BP2 exon 2, IRF2BP2-RARA fusion, and RARA intron 2 using Clustal Omega show breakpoints in exon 2 of IRF2BP2 gene and intron 2 of RARA gene.

$\mathrm{X}$ receptor $(\mathrm{RXR}) .{ }^{21}$ This RARA-RXR complex is required for promyelocyte differentiation. Various X-RARA fusion proteins could have a dominant negative effect on wild-type RARA and X proteins, or form heterodimers sequestering RXR and recruiting corepressors and histone deacetylase complex to 
Yin et al

repress genes implicated in myeloid differentiation. ${ }^{22}$ Similarly, IRF2BP2-RARA may promote leukemogenesis by serving as a dominant negative regulator of RARA and IRF2BP2. In the present case, the patient initially responded well to ATRA, arsenic trioxide, and gemtuzumab ozogamicin; therefore, APL with IRF2BP2-RARA seems to be sensitive to these drugs. However, early disease relapse suggests that APL with IRF2BP2-RARA may have a more aggressive clinical course and may require more intensive therapy.

\section{Conclusions}

This report presents a case of APL with a novel IRF2BP2-RARA fusion. The patient initially responded to ATRA, arsenic trioxide, and gemtuzumab ozogamicin; however, her disease relapsed shortly after completion of consolidation therapy, suggesting that the sensitivity to these drugs is limited and of short duration. This is the first report showing involvement of IRF2BP2 in APL, and it expands the list of novel RARA partners identified in APL. This case also shows the importance of morphologic examination to establish the diagnosis of APL based on the combination of cytologic features, strong myeloperoxidase reactivity, and a positive POD test, which led to evidence of a novel RARA fusion partner.

\section{Acknowledgments}

The authors wish to thank Dr. Roger Schultz from Signature Genomics Laboratories for his assistance with oligonucleotide microarray analysis.

\section{References}

1. Wang ZY, Chen Y. Acute promyelocytic leukemia: from highly fatal to highly curable. Blood 2008;111:2505-2515.

2. Chen Z, Brand NJ, Chen A, et al. Fusion between a novel Kruppel-like zinc finger gene and the retinoic acid receptor-alpha locus due to a variant $\mathrm{t}(11 ; 17)$ translocation associated with acute promyelocytic leukaemia. EMBO J 1993;12:1161-1167.

3. Grimwade D, Biondi A, Mozziconacci MJ, et al. Characterization of acute promyelocytic leukemia cases lacking the classic $t(15 ; 17)$ : results of the European Working Party. Blood 2000;96:1297-1308.
4. Redner RL, Rush EA, Faas $\mathrm{S}$, et al. The $\mathrm{t}(5 ; 17)$ variant of acute promyelocytic leukemia expresses a nucleophosmin-retinoic acid receptor fusion. Blood 1996;87:882-886.

5. Wells RA, Catzavelos C, Kamel-Reid S. Fusion of retinoic acid receptor alpha to NuMA, the nuclear mitotic apparatus protein, by a variant translocation in acute promyelocytic leukaemia. Nat Genet 1997;17:109_ 113 .

6. Arnould C, Philippe C, Bourdon V, et al. The signal transducer and activator of transcription STAT5b gene is a new partner of retinoic acid receptor alpha in acute promyelocytic-like leukaemia. Hum Mol Genet 1999;8:1741-1749.

7. Catalano A, Dawson MA, Somana $K$, et al. The PRKAR1A gene is fused to RARA in a new variant acute promyelocytic leukemia. Blood 2007;110:4073-4076.

8. Kondo T, Mori A, Darmanin S, et al. The seventh pathogenic fusion gene FIP1L1-RARA was isolated from a t $(4 ; 17)$-positive acute promyelocytic leukemia. Haematologica 2008;93:1414-1416.

9. Yamamoto Y, Tsuzuki S, Tsuzuki M, et al. BCOR as a novel fusion partner of retinoic acid receptor alpha in a $\mathrm{t}(\mathrm{X} ; 17)(\mathrm{p} 11 ; \mathrm{q} 12)$ variant of acute promyelocytic leukemia. Blood 2010;116:4274-4283.

10. Won D, Shin SY, Park CJ, et al. OBFC2A/RARA: a novel fusion gene in variant acute promyelocytic leukemia. Blood 2013;121:1432-1435.

11. Dimov ND, Medeiros LJ, Kantarjian HM, et al. Rapid and reliable confirmation of acute promyelocytic leukemia by immunofluorescence staining with an antipromyelocytic leukemia antibody: the M. D. Anderson Cancer Center experience of 349 patients. Cancer 2010;116:369-376.

12. Kolguist KA, Schultz RA, Furrow A, et al. Microarray-based comparative genomic hybridization of cancer targets reveals novel, recurrent genetic aberrations in the myelodysplastic syndromes. Cancer Genet 2011;204:603-628.

13. Ravandi F, Estey E, Jones D, et al. Effective treatment of acute promyelocytic leukemia with all-trans-retinoic acid, arsenic trioxide, and gemtuzumab ozogamicin. J Clin Oncol 2009;27:504-510.

14. Childs KS, Goodbourn S. Identification of novel co-repressor molecules for interferon regulatory factor-2. Nucleic Acids Res 2003;31:30163026.

15. Carneiro FR, Ramalho-Oliveira R, Mognol GP, et al. Interferon regulatory factor 2 binding protein 2 is a new NFAT1 partner and represses its transcriptional activity. Mol Cell Biol 2011;31:2889-2901.

16. Teng AC, Al-Montashiri NA, Cheng BL, et al. Identification of a phosphorylation-dependent nuclear localization motif in interferon regulatory factor 2 binding protein 2. PLos One 2011;6:e24100.

17. Koeppel M, van Heeringen SJ, Smeenk L, et al. The novel p53 target gene IRF2BP2 participates in cell survival during the p53 stress response. Nucleic Acids Res 2009;37:322-335

18. Tinnikov AA, Yeung KT, Das S, et al. Identification of a novel pathway that selectively modulates apoptosis of breast cancer cell lines. Cancer Res 2009;69:1375-1382.

19. Blotta S, Tassone P, Prabhala RH, et al. Identification of novel antigens with induced immune response in monoclonal gammopathy of undetermined significance. Blood 2009;114:3276-3284.

20. Nyguist KB, Panagopoulos I, Thorsen J, et al. Whole transcriptome sequencing identifies novel IRF2BP2-CDX1 fusion gene brought about by translocation $\mathrm{t}(1 ; 5)(\mathrm{q} 42 ; \mathrm{q} 32)$ in mesenchymal chondrosarcoma. PLos One 2012; 7:e49705

21. Melnick A, Licht JD. Deconstructing a disease: RARalpha, its fusion partners, and their roles in the pathogenesis of acute promyelocytic leukemia. Blood 1999;93:3167-3215.

22. Mistry AR, Pedersen EW, Solomon E, et al. The molecular pathogenesis of acute promyelocytic leukaemia: implications for the clinical management of the disease. Blood Rev 2003;17:71-91. 